\title{
Novel route to neuroprotection
}

Molecules with broad pro-neurogenic and/or neuroprotective properties could have therapeutic applications in a range of neurodegenerative diseases. In two related studies published in PNAS, compounds from a series based on P7C3 an aminopropyl carbazole with neurogenic properties - have been found to have therapeutic effects in both mouse and worm models of Parkinson's disease (PD) and in a mouse model of amyotrophic lateral sclerosis (ALS).

P7C3 was originally discovered in a target-agnostic in vivo screen for compounds that are able to enhance neurogenesis in the hippocampus, where it acts by blocking the death of newborn cells. In the two more recent studies, the authors investigated whether these effects would extend to adult neurons outside the hippocampus - the dopaminergic neurons of the substantia nigra that are affected in PD - and outside the brain, in this case the motor neurons in the spinal cord that are affected in ALS.

The authors used an established mouse model of PD, in which administration of the toxin MPTP (1-methyl-4-phenyl-1,2,3,6-tetrahydropyridine) selectively kills neurons in the substantia nigra, causing effects that resemble PD. After 24 hours, mice were treated with P7C3 or P7C3A20 - an analogue of $\mathrm{P} 7 \mathrm{C} 3$ that had higher potency and lower toxicity in the previous study. These compounds were also compared with Dimebon (latrepirdine), a compound that has shown neuroprotective activity in animal models of Alzheimer's disease and Huntington's disease but failed in Phase III trials. Immunohistochemical staining was used to reveal the number of dopaminergic neurons remaining in the mouse brains after MPTP treatment.

P7C3A20 offered the best protection against MPTP-induced toxicity in both mouse and worm models of PD, followed by P7C3, but Dimebon had no effect in either model. P7C3A20 had a higher ceiling of efficacy than P7C3 and a lower dose was required for a comparable effect. A high dose of P7C3A20 almost completely blocked the effect of MPTP in the mouse model. In addition, worms treated with P7C3A20 retained $80 \%$ of their usual movement after exposure to MPTP, compared to $60 \%$ for those treated with $\mathrm{P} 7 \mathrm{C} 3$ and no protective effects for those treated with Dimebon.

To explore the activity of the P7C3 series outside the brain, the authors used a mouse model of ALS expressing mutant superoxide dismutase 1 $\left(\mathrm{SOD} 1^{\mathrm{G} 93 \mathrm{~A}}\right)$. As in the PD model, P7C3A20 afforded the greatest protection against disease progression, P7C3 provided moderate protection and Dimebon had no effect.

The potency of the three compounds in the PD models and ALS model followed the same pattern observed in the earlier study on the newborn neurons of the hippocampus, and this link was also seen when more P7C3 analogues

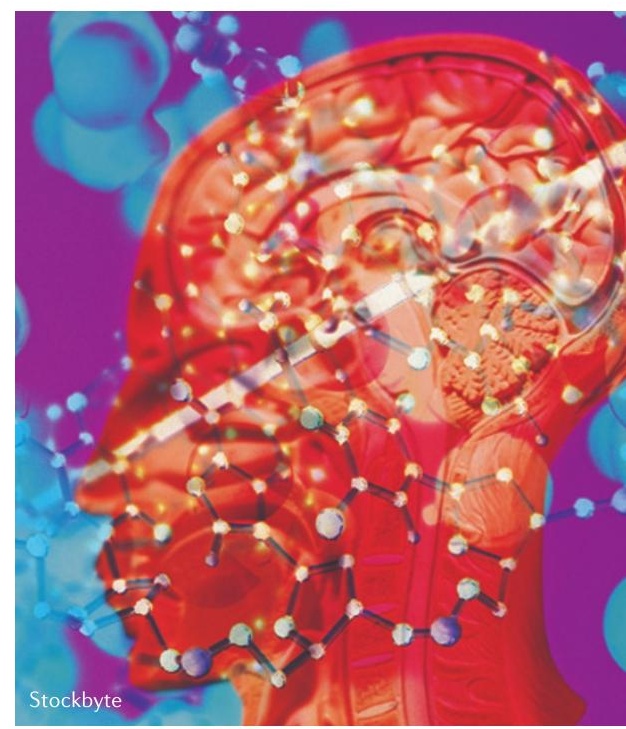

were tested. The authors therefore suggest that screening candidates for hippocampal neurogenesis activity — a relatively quick process - might speed up the identification of neuroprotective molecules. They also plan further work to optimize the properties of the P7C3 analogues to reduce toxicity and improve delivery, and suggest that P7C3 analogues could be developed into treatments for both $\mathrm{PD}$ and ALS.

Samia Burridge

ORIGINAL RESEARCH PAPERS De Jesús-Cortés, H. et al. Neuroprotective efficacy of aminopropyl carbazoles in a mouse model of Parkinson disease. Proc. Natl Acad. Sci. USA 109, 17010-17015 (2012)| Tesla, R. et al. Neuroprotective efficacy of aminopropyl carbazoles in a mouse model of amyotrophic lateral sclerosis. Proc. Natl Acad. Sci. USA 109, 17016-17021 (2012)

FURTHER READING Pieper, A. A. et al. Discovery of a proneurogenic, neuroprotective chemical. Cell 142, 39-51 (2010) 